\title{
SIMULATION THE PROCESS OF IRON REMOVAL THE UNDERGROUND WATER BY POLYSTYRENE FOAM FILTERS
}

\author{
Valeriy Orlov ${ }^{1}$, Andrii Safonyk ${ }^{2} \S$, \\ Sergiy Martynov ${ }^{3}$, Sergiy Kunytskyi ${ }^{4}$ \\ 1,2,3,4 The National University of Water and Environmental \\ Engineering, 33000, st. Soborna 11, Rivne, UKRAINE
}

\begin{abstract}
A mathematical model based on the research process by the contact groundwater iron removal filters with polystyrene upward seepage takes into account reverse influence determinants (concentration of fluid contamination and sediment) for the environment characteristics (porosity coefficient, mass exchange). Construct the algorithm of solution the regularly perturbed nonlinear task as "convection - mass exchange". On this basis, conducted a computer experiment involving experimental data confirmed the adequacy of the model.
\end{abstract}

AMS Subject Classification: 65E05, 65M25, 65M32, 68U20, 65C20

Key Words: mathematical model of the iron removal, the asymptotic decision, perturbation, polystyrene foam filter

\section{Introduction}

Human activity has a negative impact on the environment, including water resources and that leads to significant pollution [5]. In general, groundwater

\footnotetext{
Received: $\quad$ April 20, 2016

Revised: $\quad$ August 10, 2016

Published: October 7, 2016

$\S_{\text {Correspondence author }}$
}

(c) 2016 Academic Publications, Ltd.

url: www.acadpubl.eu 
compared to surface is more protected. However, only a small part of groundwater in Ukraine meets the applicable standards for drinking water [4], . Typically, most protected underground source has a high concentration of iron, due to the prevalence of this element in the earth's crust and is associated with regional, climatic, hydrological and landscape features [6]. Iron in natural waters can be in the form of bi- and trivalent ions, organic and inorganic colloids and bacterial origin. Setting the form of iron significantly affects the choice of iron removal [9]. Existing methods of iron removal of water can be divided into nonchemical, reagent, ion exchange, membrane and biochemical. The method of iron removal of water must choose tryouts iron removal of water directly from the water source [6]. For iron removal of water typically used nonchemical methods due to their relative cheapness. When iron removal of water as granular loading granular filters using natural and artificial materials: silica sand, stone, granite gravel, polystyrene, zeolite, etc. [5]. Type Filter parameters and its grain layer height significantly affect the efficiency of iron removal of water, the size of filter systems and their performance. One of the economically viable downloads is polystyrene which has a number of technical and economic benefits [4]. Fist proposed to use it as download fast filters at the Department of Water and Drilling in the National University of Water and Environmental Engineering, (NUWEE) Rivne, Ukraine). Polystyrene download is recommended for use in the preparation of potable water acting normative documents [6]. For water purification from iron ions we recommended the scheme of contact iron removal of water which is shown in Fig. 1. Underground water by pipeline 1 enters the vacuum ejection aerator 2 for the saturation of oxygen and removal of dissolved gases (hydrogen sulfide, carbon dioxide). Next aerated water gets into the air separator 3 where taking place the removal of dissolved gases in the filtered space polystyrene filter 5. Passing up flow polystyrene download 6 , held in a flooded state by special designed hold grating 7 , water cleaned of iron and going into space over the filters from here gravity is given by pipeline 8 to consumers. Filters are washed alternately with iron removal water from the combined filter space by opening the latch on the proper flushing pipe 9 Speed filtering free-flow filter is recommended to take $7 \ldots 10 \mathrm{~m} / \mathrm{h}$. Washing foam download provided downstream purified water with an intensity of $10 \ldots 18 \mathrm{l} /\left(\mathrm{s} \mathrm{m}^{2}\right)$ for $3 . . .4 \mathrm{~min}[6]$. You can embed air separator and filter in a metal tower column [7]. This can be used simplified aeration with free air separator spout water from a height of at least $0.5 \mathrm{~m}$ or reinforced vacuum aeration [4]. At the end of the water iron removal filter cycles due to the achievement of limit head losses and therefore the transfer the filter mode to the washing mode and conversely can be carried out using the device without the use of hydro-valves [6]. Wa- 


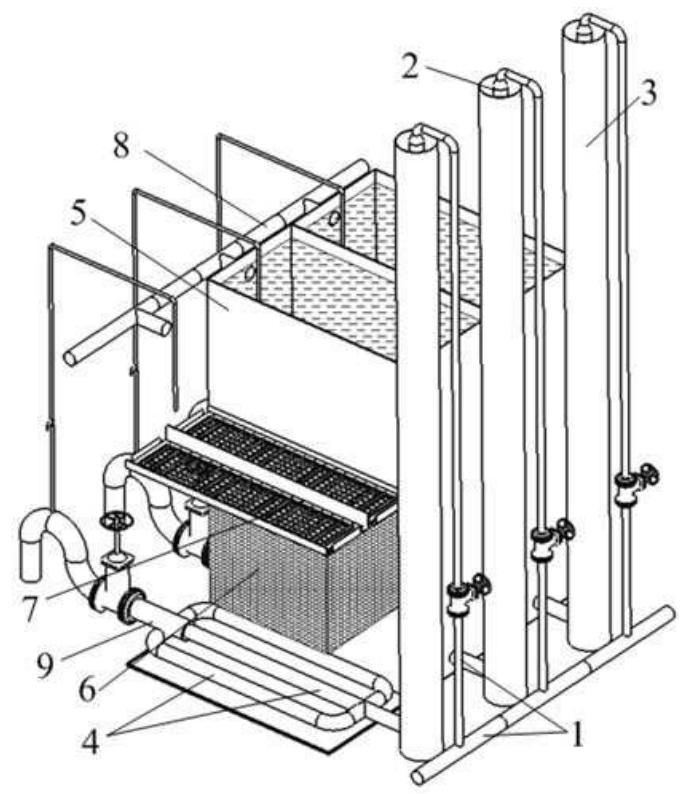

Figure 1: Scheme of iron removal of water by aeration and filtration for polystyrene filters with upward a flow filtration; 1 - pipeline underground water supply; 2 - aerator; 3 air separator; 4 - lower drainage distribution system; 5 - filter housing; 6 - polystyrene download; 7 hold grid; 8 - drainage pipe of water without iron (filtrate); 9 - wash piping

ter contact iron removal stations we introduced in Kyiv, Rivne, Khmelnytsky, Vinnytsia and other regions of Ukraine [6] and provide the concentration of iron in the filtrate of not more than $0.2 \mathrm{mg} / d \mathrm{~m}^{3}$. The choice of parameters of polystyrene download and filters generally quite a difficult task and can be solved using mathematical modeling.

\section{Mathematical Modeling Problem Formulation}

The famous analytical equations that describe the filtering process [3], do not permit a wide range of parameters describing the cleaning process, moreover, do not include the impact of the reverse characteristics of the cleaning process. Therefore, the urgent task is to develop theoretical studies of nonlinear mathematical modeling of contact iron removal of water. Consider the process of 
contact water iron removal that implement in the granular filter $(0 \leq x \leq L)$ with homogeneous filter loading, Highlighted (deformation) block allows for material balance and kinetics of the process considering the reverse effect of particles deposition on the porous $\sigma$ and mass exchange coefficients $\beta, \alpha$, and also on the filtration coefficient [1], [2], [8],

$$
\left\{\begin{array}{l}
\frac{\partial(\sigma(\rho(x, t)) c(x, t))}{\partial t}+\frac{\partial \rho(x, t)}{\partial t}+v \frac{\partial c(x, t)}{\partial x}=0 \\
\frac{\partial \rho(x, t)}{\partial t}=\beta(\rho(x, t)) c(x, t)-\varepsilon \alpha(\rho(x, t)) \rho(x, t),
\end{array}\right.
$$

where $c(x, t)$ concentration of iron compounds in the filtered liquid medium; $\rho(x, t)$ concentration of iron compounds deposited in filter downloading; $\beta(\rho)$ coefficient characterizing massive the volume of impurity deposition of particles per unit of time

$$
\beta(\rho)=\left(\beta_{0}+\varepsilon \beta_{* \rho}(x, t)\right) v^{\gamma_{1}} d^{\gamma_{2}},
$$

where $\alpha(\rho)$ coefficient characterizing the massive volumes of separated for the same time of granules download impurity particles

$$
\alpha(\rho)=\alpha_{0}+\varepsilon \alpha_{* \rho}(x, t),
$$

parameters $\beta_{0}, \alpha_{0}$ are established experimental method [6]; $v$ speed filtering $v=$ const; d equivalent diameter of granules download; $\gamma_{1}, \gamma_{2}$ empirical coefficients $[3] ; c_{*}^{*}(t)$ concentration of impurity particles inlet of filter; $\sigma(\rho)$ porous of filter downloading ( $\sigma_{0}$ initial porous download)

$$
\sigma(x, t)=\sigma_{0}-\varepsilon \sigma_{* \rho}(x, t),
$$

where $\alpha_{0}, \alpha_{*}, \beta_{0}, \beta_{*}, \sigma_{0}, \sigma_{*}, \varepsilon$ rigid parameters (they characterize the corresponding coefficients; $\beta(\rho), \alpha(\rho), \sigma(\rho)$ soft options and found by the research method); $\varepsilon$ small parameter

The solution algorithm (asymptotic). Solutions supplies (1) provided (2) searching as asymptotic series [1], [2], [8],

$$
\begin{aligned}
& c(x, t)=c_{0}(x, t)+\sum_{i=1}^{n} \varepsilon^{i} c_{i}(x, t)+R_{1}(x, t, \varepsilon), \\
& \rho(x, t)=\rho_{0}(x, t)+\sum_{i=1}^{n} \varepsilon^{i} \rho_{i}(x, t)+R_{2}(x, t, \varepsilon),
\end{aligned}
$$

where $R_{1}, R_{2}$ residual members; $c_{i}(x, t), \rho_{i}(x, t)(i=\overline{0, n})$ member of regular units of asymptotes. 
Similar to [1], [2], [8], after substituting (6) - (7) to (1) and the use of standard "equating procedures" for finding of functions $c_{i}$ and $\rho_{i}(i=\overline{0, n})$ coming to the following tasks

$$
\begin{gathered}
\left\{\begin{array}{c}
\sigma_{0} \frac{\partial c_{0}}{\partial t}+v \frac{\partial c_{0}}{\partial x}+\frac{\partial \rho_{0}}{\partial t}=0, \quad \frac{\partial \rho_{0}}{\partial t}=\beta_{0} v d c_{0}, \\
\left.c_{0}\right|_{x=0}=c_{*}^{*}(t),\left.\quad c_{0}\right|_{t=0}=0,\left.\quad \rho_{0}\right|_{x=0}=0,\left.\quad \rho_{0}\right|_{t=0}=0,
\end{array}\right. \\
\left\{\begin{array}{l}
\sigma_{*} \rho_{i-1} \frac{\partial c_{i}}{\partial t}+v \frac{\partial c_{i}}{\partial x}+\sigma_{*} \frac{\partial \rho_{i-1}}{\partial t} c_{i}+\frac{\partial \rho_{i}}{\partial t}=0, \quad \frac{\partial \rho_{i}}{\partial t}=-\beta_{*} \rho_{i-1} v d c_{i}-g_{i}, \\
\left.c_{i}\right|_{x=0}=0,\left.\quad c_{i}\right|_{t=0}=0,\left.\quad \rho_{i}\right|_{x=0}=0,\left.\quad \rho_{i}\right|_{t=0}=0, i=\overline{1, n}
\end{array}\right.
\end{gathered}
$$

As a result of their decision we have

$$
\begin{aligned}
& c_{0}(x, t)=\left[\begin{array}{l}
c_{*}^{*}\left(t-\frac{\sigma_{0} x}{v}\right) \cdot e^{-\frac{\beta_{0} x}{v}}, t \geq \frac{\sigma_{0} x}{v}, \\
0, \quad t<\frac{\sigma_{0} x}{v}
\end{array}\right. \\
& \rho_{0}(x, t)=\beta_{0} v d \int_{0}^{t} c_{0}(x, \tilde{t}) d \tilde{t} \\
& c_{i}(x, t)=\left[\begin{array}{l}
\frac{e^{-\lambda_{i}(x, t)}}{v} \cdot \int_{0}^{x} g_{i}\left(\tilde{x}, f_{i}(\tilde{x})+x-f_{i}(x)\right) \cdot e^{\lambda_{i}(\tilde{x}, t)} d \tilde{x}, \quad t \geq f_{i}(x), \\
0, \quad t<f_{i}(x),
\end{array}\right. \\
& \rho_{i}(x, t)=\int_{0}^{t}\left(-\beta_{*} v d \rho_{i-1}(x, \tilde{t}) c_{i}(x, \tilde{t})-g_{i}(x, \tilde{t})\right) d \tilde{t}
\end{aligned}
$$

where

$$
\begin{gathered}
g_{i}(x, t)=\sum_{j=1}^{i} \rho_{j-1}\left(\alpha_{0}+I(i, j) \sum_{j=2}^{i}\left(\alpha_{*} \rho_{i-2}\right)\right), \\
\lambda_{i}(x, t)=\frac{1}{v} \int_{0}^{x} \psi_{i}\left(\tilde{x}, f_{i}(\tilde{x})+x-f_{i}(x)\right) d \tilde{x}, \\
\psi_{i}(x, t)=\sigma_{*} \frac{\partial \rho_{i-1}(x, t)}{\partial t}-\beta_{* v d \rho_{i-1}}(x, t), \\
I(a, b)= \begin{cases}1, & \text { if } a \geq b, \\
0, & \text { if } a<b .\end{cases}
\end{gathered}
$$

Approximate value functions $f_{i}(x)$ finding by interpolation of array $\left(x_{j}, t_{j}\right)$, $j=\overline{1, n}$ where $x_{j}=\Delta x \cdot j, t_{j+1}=t_{j}+\frac{\Delta x}{v} \sigma_{*} \rho_{i-1}\left(x_{j}, t_{j}\right)$. For estimation of remaining members we have a similar task appropriate to [1], [2], [8], . 


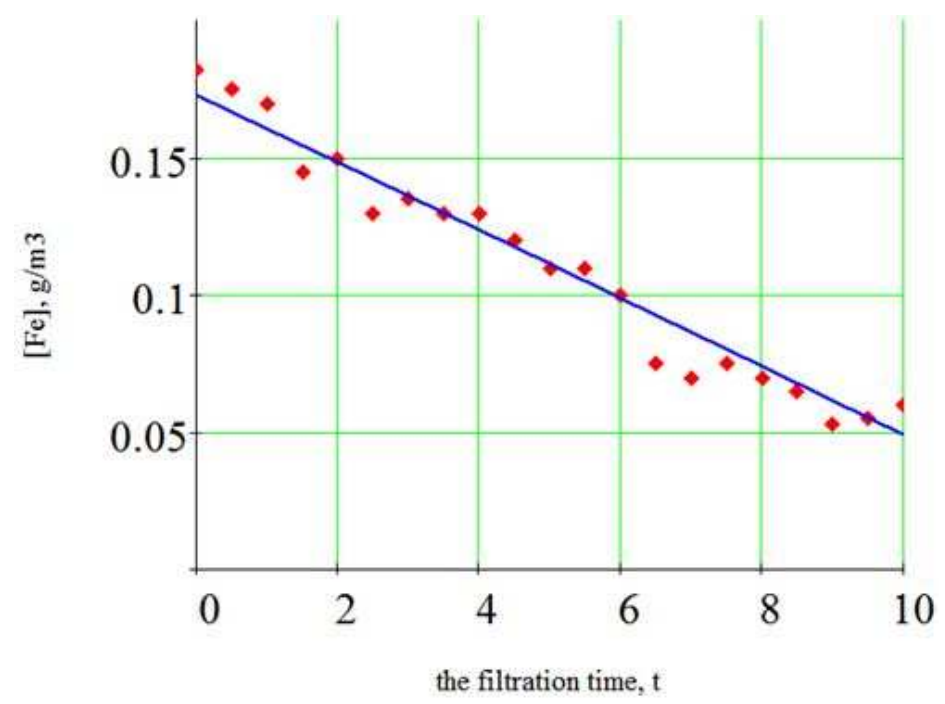

Figure 2: Changing the concentration of iron in the filtrate with time (experimental and simulated data)

\section{Numerical Calculations}

The results of the filtering process calculations based computer experiment by formulas $(6)-(7)$ at $c_{*}^{*}(t)=1 \mathrm{~g} / \mathrm{m}^{3}, \beta_{0}=3 \cdot 10^{-4} \mathrm{~s}^{-1}, \alpha_{0}=2 \cdot 10^{-7} \mathrm{~s}^{-1}$, $v=5 \mathrm{~m} / \mathrm{h}, L=1 \mathrm{~m}, \sigma_{0}=0.44 ; \sigma_{*}=1, \alpha_{*}=1, \beta_{*}=1, d=2.8 \mathrm{~mm}, \varepsilon=0.01$.

Figure 2 accounted catalytic effect of the cleaning process by modifying factor $\beta(\rho)$ (4) depending on the concentration of sediment $\rho$ in layer loading. This allows for a gradual increase in the efficiency of iron removal of water, which is confirmed by theoretical research and experimental data [6].

Figure 3 shows the distribution of pollution download height. As we see, the basic number pollution lingers at the bottom of the download.

\section{Conclusions}

The mathematical model describes patterns of detention iron hydrate in porous polystyrene filter and takes into account the reverse effect characteristics of the process (sediment concentration) for filtering parameters. The proposed algorithm solving the problem of determining the corresponding time ,protective action of the polystyrene filter. The results of calculating the distribution of 


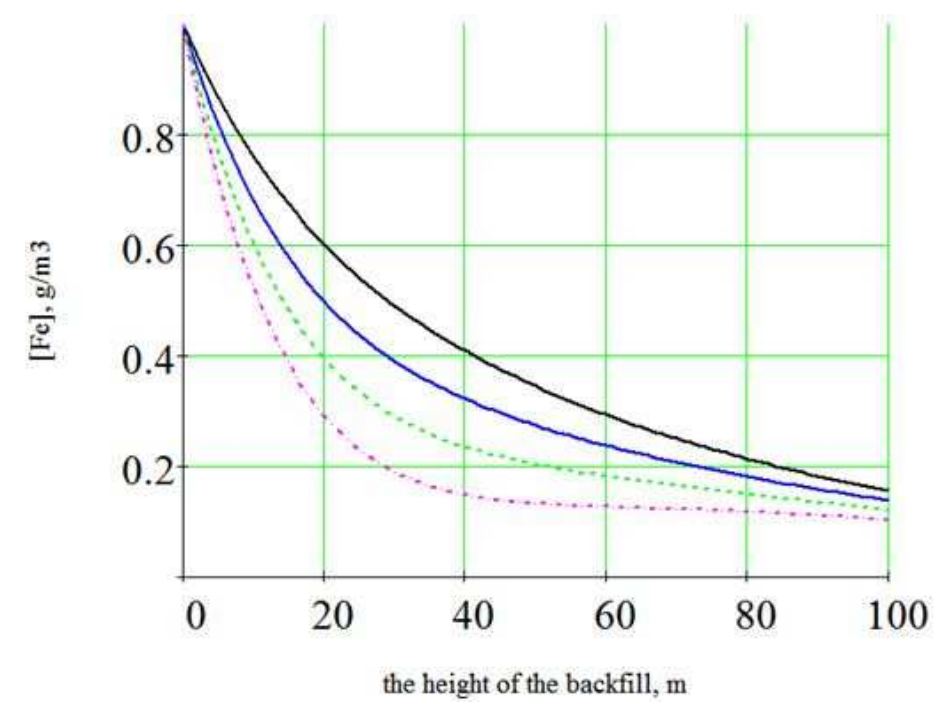

Figure 3: Changing the concentration of iron download height (from top to bottom for the time $t=2 h, t=4 h, t=6 h, t=8 h$ in accordance)

impurity concentration and mass amount of impurities filter height for different points in time, and time of protective action (filter cycle) download.

\section{References}

[1] A. Safonyk and A. Bomba (2015) Int. J. Appl. Math. Res. 4(1), 1-7.

[2] A. Bomba, A. Safonyk and E. Fursachik (2013) Journal of Automation and Information Sciences 45(4), 16-22.

[3] D. Mynts, Theoretical fundamentals of cleaning water technology, Moscow : Stroiizdat, (1964), 155.

[4] V. Orlov, S. Martynov, Contact iron removal of water on the polystyrene foam filters. Monograph, LAP LAMBERT Academic Publishing, (2015), 126.

[5] V. Orlov, S. Martynov, Clean natural water by polystyrene filters. Monograph, Moscow : Stroiizdat, (1964), 155.

[6] V. Orlov, Iron removal of underground water by simplified aeration and filtration, Rivne : NUWEE, (2008), 158.

[7] V. Orlov, S. Martynov, N. Minaev, L. Odud, K. Mamchur Patent 77541 Ukraine. IPC C $02 \mathrm{~F} \mathrm{1/64,} \mathrm{B} 01 \mathrm{D} 24 / 02$. Tower column of polystyrene-silicon filter the applicant and patentee National University of Water and Environment Engineering. u 2012 06084; appl. 21.05.12; publ. 02.25.13, Bull. 4. 
[8] 12. A. Safonyk (2015) Int. J. Math. Models and Methods in Appl. Sciences 9, 189-192.

[9] V.Stankyavychus, Deironing water filtration (the basic theory and calculation units), Vilnius: Mokslas, (1978), 120. 